\title{
Grundrauschen im Prokrustesbett: Zur Umsetzung des neuen Entgeltgesetzes in der Psychiatrie
}

\author{
Background Noise in the Procrustean Bed: Implementation at a New Hospital \\ Reimbursement System in Germany
}

Autor

Institut
Ingrid Munk

Klinik für Psychiatrie, Psychotherapie und Psychosomatik, Vivantes Klinikum Neukölln

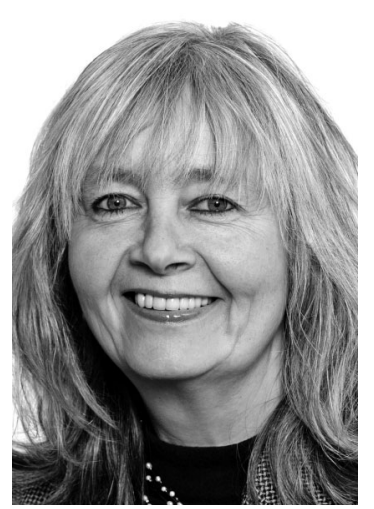

Dr. Ingrid Munk
Für nicht in der antiken Mythologie Kundige: Der Räuber Prokrustes nahm Menschen gefangen. Große Menschen fesselte er an ein kleines Bett und hackte ihnen die Füße ab; kleine Menschen streckte er auf dem Bett so lang, bis es passte. Im übertragenen Sinne steht das Prokrustesbett für Versuche, einem lebendigen, gewachsenen System ein unpassendes aufzuzwingen. Zunächst sah es in der Tat so aus, als ob dies mit vereinten Kräften aller maßgeblichen Player [1] beim neuen Entgeltgesetz verhindert werden könnte: DRGs sind wegen Nichteignung zur Abbildung der Leistungen des Faches Psychiatrie, Psychotherapie und Psychosomatik nicht die Grundlage des neuen Abrechnungssystems geworden. Stattdessen ist im $§ 17 d$ des Krankenhausentgeltreformgesetzes festgehalten: Es soll ein durchgängiges, leistungsorientiertes, pauschalierendes Vergütungssystem auf der Grundlage von tagesbezogenen Entgelten entwickelt werden. Dabei soll geprüft werden, ob für bestimmte Leistungsbereiche andere Abrechnungseinheiten sinnvoll und ob die Leistungen der psychiatrischen Institutsambulanz einzubeziehen sind. Diese im Gesetz formulierten Prüfaufträge sind bisher nicht erteilt worden, obwohl genau das von allen Fachverbänden gefordert wird [1]. Noch gravierender ist allerdings, dass ein Abrechnungssystem auf der Grundlage von OPS-Codes gewählt wurde, das, in psychosomatischen Reha-Kliniken gebräuchlich, zur Erfassung der Arbeitsabläufe in der Akutpsychiatrie, ähnlich den DRGs, schlicht nicht tauglich ist.

Doch zunächst ein Blick zurück: Es schien der Gesundheitspolitik nicht mehr vertretbar, Pflegesätze zwischen ca. 150 bis über $300 €$ und Verweildauern zwischen 15 und 30 Tagen, schwankend je nach Bundesland und Klinik, zu akzeptieren. So war es ihr erklärtes Ziel, die Klinikbudgets aus Gründen der Gerechtigkeit anzugleichen. Eine Umverteilung reichte dem Bundesgesundheitsministerium allerdings verständlicherweise nicht: Die künftige Erlösverteilung sollte leistungsgerecht und transparent erfolgen. Vor allem aber sollten Impulse für eine Weiterentwicklung der Psychiatrie zu neuen Versorgungsformen gesetzt werden.

Jetzt ist die Umsetzungsphase im Gange. Oder sollte man besser sagen: Sie kommt auf die Kliniken zu? Rollt über sie hinweg? Während die Inhalte des Gesetzes durchaus begrüßt wurden, ist mit Art und Form der Umsetzung kaum einer zufrieden. Selbst das Institut für das Entgeltsystem im Krankenhaus (InEK), das für sich in Anspruch nimmt, bisher noch alles, was an Leistungen im Krankenhaus erbracht wird, in eine codierfähige Form gebracht zu haben, konzediert, dass die Leistungs- und Kostenaufstellungen der PretestHäuser bisher nur ca. 15-20\% des Aufwandes erfassen; eine Kalkulation ist damit nicht möglich [2]. Auch die Krankenkassen äußern sich zunehmend skeptisch und distanzieren sich von dem Projekt. Die Fachgesellschaft DGPPN [3], die Aktion Psychisch Kranke [4,5], der Arbeitskreis Psychiatrischer Chefärzte an Allgemeinkrankenhäusern [6], die Bundesdirektorenkonferenz [7] und die Bundesärztekammer [8] haben ebenfalls ihre Einwände formuliert.

Diese breite Skepsis sollte für alle Entscheidungsträger Anlass sein, innezuhalten und nachzudenken. Das Konstrukt, das die Verantwortung für die Gestaltung des Entgeltgesetzes vom Bundesministerium für Gesundheit an die Selbstverwaltungspartner sowie das InEK und das Deutsche Institut für Medizinische Dokumentation und Information delegiert, führt zu einer Entscheidungs- und Verantwortungsdiffusion unter den Beteiligten. Als Ergebnis dieser vielfältigen Abstimmungsprozesse auf verschiedenen Ebenen droht ein Konglomerat, das so keiner gewollt hat und - sachgerechte Vernunft bei allen Beteiligten voraussetzend - keiner wollen kann. Alle Beteiligten formulieren Unzufriedenheit mit den jeweils anderen. So spielt das InEK den Ball den Kliniken 
zu: Es müssten endlich von den Kliniken bessere Modelle zur Aufwands- und Leistungserfassung vorgelegt werden. Berücksichtigt wird allerdings nicht, dass, je kleinteiliger die Leistungserfassung ist, auch der Dokumentationsaufwand zunimmt, bis dahin, dass eine Dokumentation kurzzeitiger Leistungen, quasi in Echtzeit, vermutlich 20-25\% der Arbeitszeit in Anspruch nehmen würde - in einem Fach wie der Psychiatrie, das von abgestimmten, kurzen, patientenzentrierten Interventionen lebt. Zusätzlicher Dokumentationsaufwand ist nicht gerade motivationsfördernd für die Mitarbeiter. Er bindet Arbeitszeit, die für den Kontakt mit dem Patienten nicht mehr zur Verfügung steht.

Das Bundesministerium für Gesundheit hat die Erwartung an die Selbstverwaltungspartner formuliert, eine zügige Kalkulation durchzuführen, um dann Relativgewichte und aufwandsgewichtete Tagespauschalen festlegen zu können, wie bei den somatischen DRGs, und moniert, dass noch keine Kostentrenner identifiziert sind.

Die Kliniken kritisieren die simple Übertragung der DRG-Logik durch das InEK, können aber, in welcher Form auch immer, Mathematikern und Betriebswirten nicht verständlich machen, worin die Inhalte ihrer Arbeit und die Leistungen der Mitarbeiter auf psychiatrischen Stationen bestehen. So berichtete ein PretestHaus, dass das InEK kostenintensive Patienten mit 24 Stunden 1:1-Betreuung aus der Kalkulation nehmen wollte mit der Begründung, es müsse sich um „Ausreißer“ handeln. Auch die regionale Versorgungsverantwortung mit Aufnahmeverpflichtung und Notfallversorgung ist bisher nicht in der Kalkulation berücksichtigt $[8,9]$.

Schon jetzt zeichnet sich deutlich ab, dass die zurzeit gültigen OPS-Codes nicht die bezüglich der Kosten unterscheidbaren Patientengruppen liefern; es besteht sogar ein inverser Zusammenhang zwischen Schwere der Erkrankung und Anzahl der Therapieeinheiten. Nichtsdestotrotz sehen wir auf Tagungen die ersten Folien mit Aufstellungen der Krankenkassen, wie viele Therapieeinheiten je Behandlungstag erbracht wurden, wie viele davon von Ärzten, verbunden mit der süffisanten Feststellung, es könne doch nicht sein, dass ein Arzt in der Psychiatrie im Durchschnitt nicht einmal eine Therapieeinheit am Tag erbringt. Dies wird jedoch weniger Mängeln des Erfassungssystems zugeschrieben, sondern es werden Zweifel an der Effektivität psychiatrischer Arbeit gesät - obwohl gleichzeitig alle Beteiligten konzedieren, dass die OPS-Codes wohl keine adäquate Leistungserfassung darstellen.

Von Anfang an legte sich das InEK fest, dass die Einteilung nach PsychPV-Bereichen (Pseudo-OPS) keine Kostentrenner liefern würde - und bleibt dabei, ohne die vorliegenden Daten durchzurechnen. Mittlerweile sieht das InEK zwar auch, dass die OPSCodes keine Kostentrenner liefern und fordert die Kliniken auf, neue und bessere Leistungserfassungssysteme für die Kalkulation zu entwickeln. Trotzdem müssen alle Kliniken weiter zwei konkurrierende Leistungserfassungen nebeneinander durchführen: Die Dokumentation der OPS-Codes und die tägliche PsychPV-Einstufung als Pseudo-OPS-Codes. Bisher hat niemand den Aufwand erfasst, der jetzt schon in den Kliniken dafür notwendig ist. Aus eigener Erfahrung kann ich sagen, dass dies für eine Station von 20 Betten 1-2 Stunden Oberarzt-Arbeitszeit pro Woche kostet, die Erfassung der Einzel- und Gruppenleistungen noch gar nicht eingerechnet. Die Leistungserfassung wird zum zentralen Thema, hinter dem das, was auf einer psychiatrischen Station praktisch geschieht und was wichtig ist, in den Hintergrund tritt. Die meisten derjenigen, die jetzt über Leistungserfassung und das Entgeltgesetz bestimmen, haben nie eine psychi- atrische Station betreten. Dies soll nicht deren Entscheidungsbefugnis infrage stellen, sondern nur auf deren begrenzte Expertise hinweisen. Das InEK sammelt, wie es selbst bekundet, nur Daten - es fühlt sich insbesondere für Inhalte nicht verantwortlich. Nichtsdestotrotz verhält es sich wie der Herr des Geschehens, der Kliniken zu - manchmal kritisierten, manchmal umworbenen - Zuarbeitern und Datenlieferanten macht.

Dies ist Ausdruck politischer Prozesse, die sukzessive gesellschaftliche Institutionen in betriebswirtschaftlich gesteuerte Organisationen transformieren wollen - verbunden mit dem Anspruch, die Abläufe in diesen Institutionen adäquat abzubilden. Damit geht auch einher, dass Leistungen und Qualität über Daten und Indikatoren vergleichbar gemacht werden sollen. Benchmark ist das Zauberwort [10]. Nicht nur Krankenhäuser sind jetzt Unternehmen, jede einzelne Station wird zum kleinen Unternehmen, dessen Leistungen erfasst, verglichen und zu den Kosten in Beziehung gesetzt werden. Die Realität auf den Stationen lässt sich bisher nicht in dieses System hineinzwängen, sodass, wie oben ausgeführt, nur 15-20\% der Kosten zuzuordnen sind. Das, was sich nicht abbilden lässt, wird im InEK-Jargon lapidar zur „Residualgröße“ oder zum „Hintergrundrauschen“ marginalisiert. Damit ist das Kernstück psychiatrischer Akutbehandlung, die Milieutherapie in der therapeutischen Gemeinschaft, in der betriebswirtschaftlichen Realität nicht vorhanden. Nicht nur das: Trotz der Behauptung, die Codierung erfasse die Leistung, droht das Umgekehrte: das, was erfasst und codiert (und in der Konsequenz bezahlt) wird, konstituiert und bestimmt das Leistungsgeschehen. So stellen trotz der gegenläufigen Appelle der Fachgesellschaften bereits jetzt Kliniken ihre Therapien auf durch 25 teilbare Zeiteinheiten um.

Die Einwände derjenigen, die Inhalte und Abläufe auf psychiatrischen Stationen kennen, werden entwertet mit der saloppen Begründung, die somatischen Kliniken hätten vor Einführung der DRGs genauso gebremst und die Psychiatrie wolle sich nur dem Fortschritt entziehen. Kritische Experten werden so zu einer Art Dinosaurier diskreditiert, die die Zeichen der Zeit noch nicht erkannt haben. Eine breite Koalition von Bundesgesundheitsministerium, Deutscher Krankenhausgesellschaft und Krankenkassen wird sichtbar, die vehement den Erfolg der DRGs in der Somatik mit dem Argument beschwört, es sei alles leistungsgerechter und transparenter geworden. Erstaunlich ist allerdings, wie wenig die eigene Forderung, die DRG-Entwicklung habe in Form eines „lernenden Systems“ zu geschehen, umgesetzt wurde, wie wenig die Veränderungen, die im Krankenhausbereich und der Gesundheitsversorgung durch die DRG-Einführung angestoßen wurden, systemisch und systematisch betrachtet wurden. Wie ist die Kosten- und Leistungsentwicklung? Welche Anreize werden im System gesetzt und ist beispielsweise die Zunahme von Herzkatheteruntersuchungen und Kniespiegelungen medizinisch und ökonomisch sinnvoll? Hier zeigt sich eine bedauerliche Weigerung, die Folgen gesundheitspolitischer Interventionen kritisch und selbstreflektiv zu verfolgen. So ist die Begleitforschung zur Einführung der DRGs nie durchgeführt worden und auch jetzt hat die Umsetzung des Entgeltgesetzes für Psychiatrie und Psychosomatik schon begonnen, ohne dass die entsprechende, im Gesetz geforderte Forschung „,begleitend“ durchgeführt wird.

Die Front aus Krankenkassen, der Deutschen Krankenhausgesellschaft und dem InEK beginnt allerdings zu wanken: Die Krankenkassen kommen langsam zu der, vermutlich realistischen, Einschätzung, dass die Spirale mehr Betten - mehr Kosten durch das neue Entgeltgesetz nicht etwa eingedämmt, sondern eher 
noch angeheizt wird. Dies ist selbstverständlich nicht das Interesse der Kostenträger.

Kurzfristig hoffe ich deshalb auf eine Koalition der Vernunft, welche ...

- die OPS-Codierung wegen Nichttauglichkeit abschafft,

- die bundesweit erfasste tägliche PsychPV-Einstufung als Grundlage eines Abrechnungssystems überprüft,

- eine fachliche fundierte Begleitforschung durchführen lässt,

- eine unabhängige Kommission zur Bewertung der bisherigen Umsetzung und der zu erwartenden Steuerungs- und Anreizsysteme einsetzt,

- die Prüfaufträge des $\S 17 d$ einbezieht, damit das Entgeltgesetz als Motor neuer, patientenzentrierter Versorgungsformen mit dem Ziel der Ambulantisierung wirksam werden kann.

Das entscheidende Qualitätskriterium des neuen Entgeltgesetzes ist für mich, inwieweit es die Türen für eine zukunftsweisende psychiatrische Versorgung öffnet und inwiefern es in der Umsetzung einfach, übersichtlich und unaufwendig ist - damit Zeit für die therapeutische Arbeit bleibt und weder die Entwicklung der Beziehung zum Patienten noch die multiprofessionelle Teamarbeit durch bürokratische Vorgaben behindert wird.

\section{Literatur}

1 Konzept für ein zukünftiges Entgeltsystem der Krankenhausbehandlung in Psychiatrie, Psychotherapie und Psychosomatik für alle Altersgruppen, „13-Verbände-Papier“; 31. Januar 2007

2 Belling $R$. Psych-Entgeltsystem: Psych-Pretest offenbart Diskussionsbedarf; führen und wirtschaften im Krankenhaus. führen \& wirtschaften im Krankenhaus 2011; 28: 61-65

3 Entgeltentwicklung in Psychiatrie und Psychosomatik. Stellungnahme der Deutschen Gesellschaft für Psychiatrie, Psychotherapie und Nervenheilkunde (DGPPN). Nr. 6/25.06.2010
4 Neuordnung der Finanzierung psychiatrisch-psychotherapeutischer Krankenhausbehandlung (Umsetzung des KHRG); Verlautbarung der AKTION PSYCHISCH KRANKE Vereinigung zur Reform der Versorgung psychisch Kranker e.V.; 12.12 .10

5 Kunze H, Schepker R. Das Ergebnis des OPS-Verfahrens für 2011 ist nicht zielführend? Memorandum der AKTION PSYCHISCH KRANKE Vereinigung zur Reform der Versorgung psychisch Kranker e.V. Januar 2011

6 Mitteilungen des Arbeitskreises der Chefärzte und Chefärztinnen von Kliniken für Psychiatrie und Psychotherapie an Allgemeinkrankenhäusern in Deutschland (ACKPA). Zur Entwicklung des neuen Vergütungssystems für psychiatrische und psychosomatische Einrichtungen nach §17d KHG 30.4.2010. Psychiat Prax 2010; 37: 260-263

7 Zehn-Thesen-Papier der BDK zum Neuen Entgeltsystem in Psychiatrie und Psychotherapie; Bundesdirektorenkonferenz; Verband leitender Ärztinnen und Ärzte der Kliniken für Psychiatrie und Psychotherapie (BDK) e.V.; BDK-Vorstand-Entgeltkommission 23.12.2010

8 Vorläufige Stellungnahme der Bundesärztekammer zur weiteren Ausgestaltung der Rahmenbedingungen der Einführung eines pauschalierenden Entgeltsystems für psychiatrische und psychosomatische Einrichtungen gemäß §17 d Krankenhausfinanzierungsgesetz (KHG) (Anfrage des Bundesministeriums für Gesundheit vom 03.01.2011), Berlin: 15. März 2011

9 Regionale Verantwortung als Basis für ein zukunftsfähiges Entgeltsystem für die Psychiatrie und Psychotherapie; Netzwerk „Steuerungsund Anreizsysteme für eine moderne psychiatrische Versorgung“; Stand 09.01.2011

10 Klimke A, Engfer R, Bauer M. Ein neues Entgeltsystem für Psychiatrie und Psychosomatik - Chance auf gerechtere Vergütung oder Einstieg in den Ausstieg aus der regionalen Vollversorgung? Psychiat Prax 2010; 37: 92-98

11 Kuhl H-C, Krneta D, Warnke I et al. Freiwilliges Benchmarking der Psychiatrischen Universitätskliniken Basel, Bern und Zürich; Methodisches Vorgehen und Erfahrungsbericht. Psychiatrie \& Neurologie 2008; 1 : $37-40$ 